\title{
Genetic stability of Aedes aegypti populations following invasion by wMel Wolbachia
}

\author{
Meng-Jia Lau ${ }^{1 *}$, Thomas L. Schmidt ${ }^{*}$, Qiong Yang ${ }^{1}$, Jessica Chung ${ }^{1,2}$, Lucien Sankey ${ }^{1}$, Perran A. Ross ${ }^{1}$ and
} Ary A. Hoffmann ${ }^{1 *}$

\begin{abstract}
Background: Wolbachia wMel is the most commonly used strain in rear and release strategies for Aedes aegypti mosquitoes that aim to inhibit the transmission of arboviruses such as dengue, Zika, Chikungunya and yellow fever. However, the long-term establishment of wMel in natural Ae. aegypti populations raises concerns that interactions between Wolbachia wMel and Ae. aegypti may lead to changes in the host genome, which could affect useful attributes of Wolbachia that allow it to invade and suppress disease transmission.
\end{abstract}

Results: We applied an evolve-and-resequence approach to study genome-wide genetic changes in Ae. aegypti from the Cairns region, Australia, where Wolbachia wMel was first introduced more than 10 years ago. Mosquito samples were collected at three different time points in Gordonvale, Australia, covering the phase before (2010) and after (2013 and 2018) Wolbachia releases. An additional three locations where Wolbachia replacement happened at different times across the last decade were also sampled in 2018. We found that the genomes of mosquito populations mostly remained stable after Wolbachia release, with population differences tending to reflect the geographic location of the populations rather than Wolbachia infection status. However, outlier analysis suggests that Wolbachia may have had an influence on some genes related to immune response, development, recognition and behavior.

Conclusions: Ae. aegypti populations remained geographically distinct after Wolbachia wMel releases in North Australia despite their Wolbachia infection status. At some specific genomic loci, we found signs of selection associated with Wolbachia, suggesting potential evolutionary impacts can happen in the future and further monitoring is warranted.

Keywords: Wolbachia, Aedes aegypti, Evolve-and-resequence, Population replacement, Genome-wide association study

\footnotetext{
*Correspondence: mengjial2@student.unimelb.edu.au; tom.

schmidt@unimelb.edu.au; ary@unimelb.edu.au

1 Pest and Environmental Adaptation Research Group, Bio21 Institute and the School of BioSciences, The University of Melbourne, Parkville, Victoria, Australia

Full list of author information is available at the end of the article
}

\begin{abstract}
Background
Wolbachia are bacteria that live inside the cells of many insects and induce important phenotypic effects on their hosts that can be harnessed for pest and disease control. Wolbachia-infected Aedes aegypti mosquitoes have now been released in multiple locations of the world [1-3] to help reduce the transmission of arboviruses such as dengue, Zika, Chikungunya and yellow fever [4-6]. Wolbachia $w \mathrm{Mel}$, which was transferred artificially from Drosophila melanogaster into Ae. aegypti [6], was first
\end{abstract}


released in Gordonvale and Yorkeys Knob, Queensland, Australia, around a decade ago [7] where it invaded the local population through cytoplasmic incompatibility (CI). CI results in uninfected females less likely to produce viable offspring if they mate with infected males. In contrast, infected females produce viable offspring when they mate with uninfected males or males infected by the same Wolbachia strain, and these offspring are infected [8]. This allows Wolbachia to invade and be self-sustained in a population but may also increase population divergence because it can reduce the "effective migration rate" $[9,10]$ between infected and uninfected populations. Wolbachia can also impact mitochondrial DNA (mtDNA) variation through indirect linkage disequilibrium [11-13].

With the success of Wolbachia in suppressing dengue following invasion [1,3], many studies have now focused on the sustainability of this approach beyond the initial spread, such as the maintenance of high infection levels [14], fitness costs $[15,16]$ and evolutionary adaptation $[17,18]$. The potential evolutionary changes in Wolbachia wMel-infected Ae. aegypti as well as in the bacterial genome itself following releases in the field have raised concerns about the long-term effectiveness of the strategy. The genetic background of the mosquito host can affect the capacity of Wolbachia to invade populations and suppress arboviruses $[2,19,20]$. Aedes aegypti has a short generation interval of about one month, and so if the introduction of Wolbachia triggers an evolutionary process in the mosquito genome this could be observable within a few years after invasion. Evolutionary changes in response to natural Wolbachia infections have previously been noted and appear to involve both the Wolbachia and host genomes [21, 22], affecting the population dynamics of Wolbachia infections. Adaptations can occur to counter any negative fitness effects of Wolbachia, as documented in Drosophila [21, 23], and negative fitness effects are particularly evident in novel infections transfected into new hosts [24].

There are no published studies investigating evolutionary changes in wild host populations at the genomic level following a Wolbachia release. Any putative changes may guide further phenotypic comparisons based on the types of candidate genes identified. However, in Ae. aegypti, there are challenges in characterizing genome changes after Wolbachia wMel infection. First, the genome of Ae. aegypti contains a large proportion (47\%) of transposable elements (TEs), which result in a large genome size (1.38Gb) compared to other mosquitoes [25-27]. TEs might also enhance rates of evolution, given that they are involved with gene regulation, and increase genome plasticity [28]. Moreover, other environmental factors in field-collected samples may be important, and the impacts of gene flow following the activity of Wolbachia release will increase the difficulty of outlier analysis. Finally, compared to model organisms, the genome of Ae. aegypti is still relatively poorly annotated, with only 256 proteins $(<1 \%)$ reviewed in the Swiss-Prot database (https://www.uniprot.org/taxonomy/7159).

In this study, we analyze pooled whole genome sequencing (WGS) data of mosquitoes from Gordonvale, Australia, where releases first took place, covering three different time points from the pre- and post-release phase. As a comparison, we also sequenced samples from the nearby Cairns area in Edge Hill, Redlynch and Yorkeys Knob, where Wolbachia replacement happened at different times across the last decade. In these locations, the population released involved a $w \mathrm{Mel}$ transinfected strain that had been repeatedly backcrossed to a Cairns field population background, with the expectation that the released background would be $99.9 \%$ Cairns [7]. For releases in Yorkeys Knob and sites around Cairns, we did not expect the release population to differ from the background population because there is movement of mosquitoes around this area as reflected by the occasional detection of Wolbachia-infected mosquitoes after the release [7]. On the other hand, Gordonvale is a relatively isolated population which may have its own seasonal dynamics. We combined analyses of temporal and spatial variation in Wolbachia infection status to reveal genetic diversity in the mosquito populations and the potential impact of Wolbachia wMel on the genome of its host Ae. aegypti.

\section{Results}

\section{Genetic variation in Aedes aegypti populations}

Aedes aegypti were collected from four sites around Cairns, Australia, at different times pre- and post-Wolbachia releases (Fig. 1, Table 1). We investigated patterns of genetic variation within populations and obtained Tajima's pi (nucleotide diversity $\pi$ ) for each population at $10 \mathrm{kbp}$ non-overlapping windows. Differences in $\pi$ among populations depended on chromosomal location. Near the center of each chromosome, diversities were low and similar for all populations (Fig. 2). In areas far from the centromere, variation between populations was large. This pattern has been observed in previous sequencing studies $[27,29]$, which may result from a high proportion of TEs and satellites in non-coding regions. Overall, nucleotide diversity was highest on chromosome 1 , which contains the sex determining locus and contains relatively lower gene densities and TEs but higher satellites compared to chromosomes 2 and 3 [30].

We calculated Tajima's D for each population at 10 kbp non-overlapping windows and at the gene level. We found that the density distributions of values were 


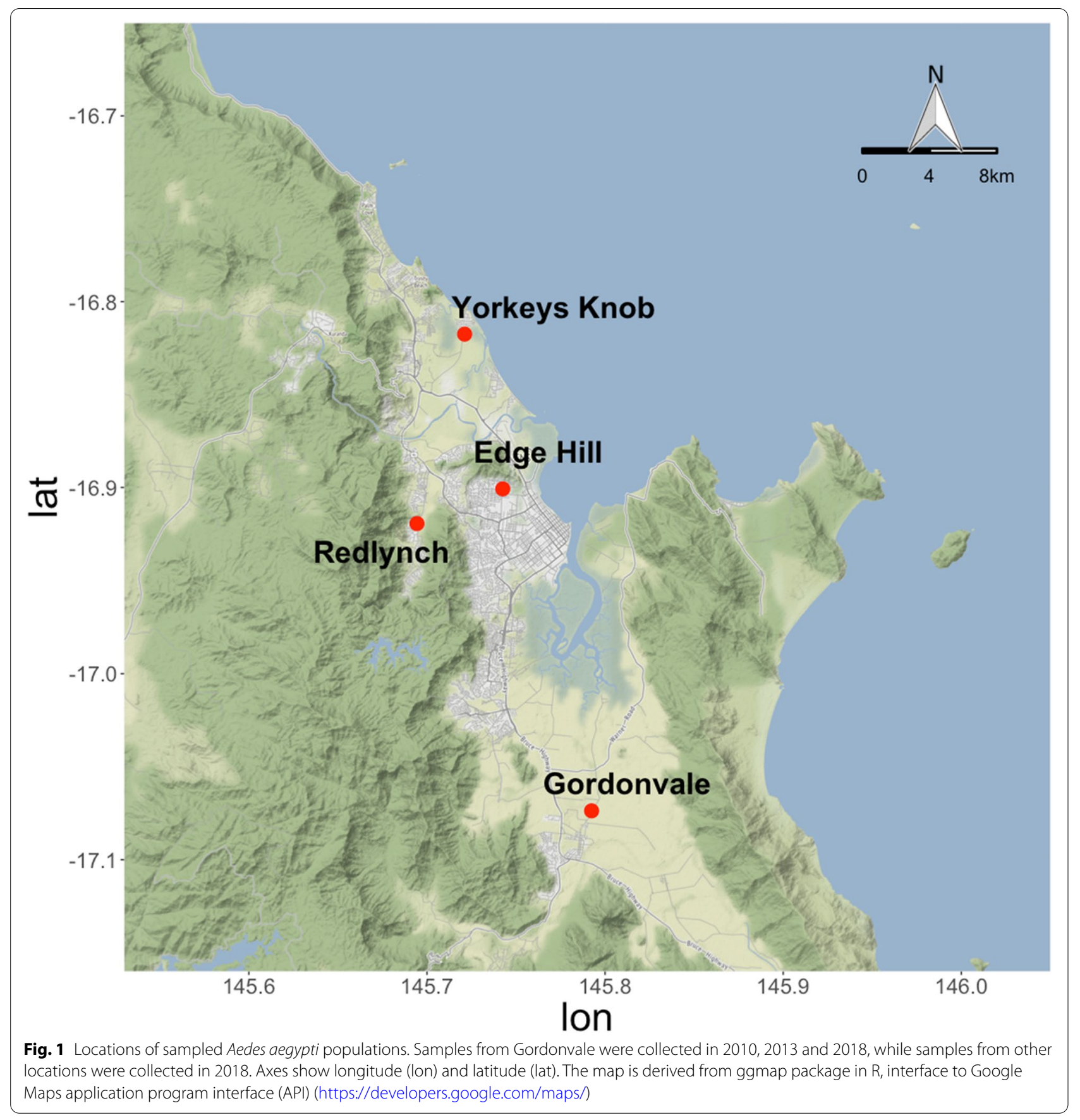

Table 1 Summary of Aedes aegypti collections and designations of samples used in comparisons

\begin{tabular}{llllll}
\hline Population name & Collecting year & Location & $\begin{array}{l}\text { Wolbachia infection } \\
\text { (Y/N) }\end{array}$ & $\begin{array}{l}\text { Year of population } \\
\text { replacement }\end{array}$ & $\begin{array}{c}\text { Sample size } \\
\text { GV10 }\end{array}$ \\
GV13 & 2010 & Gordonvale & N & 2011 & 56 \\
GV18 & 2013 & Gordonvale & Y & 2011 & 51 \\
EH & 2018 & Gordonvale & Y & 2011 & 52 \\
RL & 2018 & Edge Hill & Y & 2013 & 52 \\
YK & 2018 & Redlynch & N & 2019 & 52 \\
\hline
\end{tabular}




similar between populations, except for the Gordonvale pre-release (GV10) sample which shows a high proportion of negative values (Fig. 3). This pattern is more obvious at the gene level (Fig. 3b) than at the genome level measured in $10 \mathrm{kbp}$ non-overlapping windows (Fig. 3a), Additional file 1). The four 2018 populations converge regardless of their Wolbachia infection status or time since Wolbachia was invaded. This suggests that the pattern reflects a difference in GV10 before release rather than an effect of Wolbachia per se.

\section{Geographic segregation of Aedes aegypti populations}

We investigated genetic distances from the average of pairwise Fst (Fixation index) values through $100 \mathrm{kbp}$ nonoverlapping windows. The temporally-separated Gordonvale samples tended to have lower pairwise Fst than population pairs from different locations (Table 2). For isolation by distance (IBD) test, we also obtained a geographic distance matrix with the four 2018 populations, which was built based on the natural log transformation of the shortest road distance between the sampled locations (Table 2). Genetic distance had a weak but non-significant correlation with natural log transformed geographic distance in a Mantel test $(\mathrm{r}=0.66, p=0.12)$.

There were 461,067 single-nucleotide polymorphisms (SNPs) left after filtering with minimal depth of 50 in all populations and an average minor allele frequency $(\mathrm{MAF})>0.1$. A principal components analysis (PCA) based on these SNPs showed the first two PCs accounted for 24.2 and $22.9 \%$ of the variance respectively (Fig. 4a). The three temporally-separated Gordonvale samples fell out together, but GV10 was closer to GV18 than to GV13. We also ran PCAs on pairwise Fst differences in $100 \mathrm{kbp}$ non-overlapping windows across the genome (Fig. 4b, d) and at the gene level (Fig. 4c, e). When testing the similarity of infected or uninfected populations (Fig. 4d, e), we found little evidence for any clustering of populations related to Wolbachia infection status either across the genome or at the gene level. On the other hand, these populations clustered geographically (Fig. 4b,c). Comparisons between the Gordonvale samples and Yorkeys Knob, Edge Hill, and Redlynch showed consistent clustering by location, and stronger clustering at the gene level (Fig. 4c) than across the genome (Fig. 4b).

\section{Bayesian models to identify outliers potentially associated with Wolbachia}

To investigate potential selection associated with Wolbachia, we used 461,067 SNPs from the above filtering process and used two Bayesian models from BayPass v. 2.2 [31] for Wolbachia-related outlier analysis.

In the covariate model of BayPass, with Wolbachia infection status in the comparison, we found 2415 SNPs showing a "substantial" signature of selection with an average $\mathrm{BF}^{*}$ (Bayes Factor (BF) in $\mathrm{dB}$ units) $>5$, and 391 showing "strongly-selected" signature of selection with an average $\mathrm{BF}^{*}>10$ (Fig. 5).

The introduction of linear relationships in the covariate model, however, can result in a high false positive rate from sampling noise, particularly when large environmental effects are involved and small number of populations are compared. We therefore used a second model to calculate $\mathrm{X}^{\mathrm{T}} \mathrm{X}$, which was analogous to Fst $[31,32]$, then combined these two models to identify outliers potentially associated with Wolbachia infection.

We found that 950 (prior probability: 0.74 in each Mbp) of the "substantial" SNPs fell into the intersection of top $10 \% \mathrm{X}^{\mathrm{T}} \mathrm{X}$ values in the comparisons between GV10 and GV13 and between GV10 and GV18, including 229 SNPs distributed on chromosome 1, 390 on chromosome 2 and 330 on chromosome 3, while one was found on an assembly scaffold NW_018735222.1. A proportion of these SNPs were concentrated in specific regions, with posterior probability at least five times greater than prior probability (>3.7 in each $\mathrm{Mbp}$ ), suggesting selection due to Wolbachia (Table 3). These SNPs were considered as "substantial" outliers associated with the Wolbachia infection.

We also used a stricter criterion: average $\mathrm{BF}^{*}>10$ and falling into the intersection of top $5 \% \mathrm{X}^{\mathrm{T}} \mathrm{X}$ values in the GV10, GV13 comparison and the GV10, GV18 comparison. We then found 113 SNPs that were highly associated with Wolbachia infection and were considered as "strong" outliers.

Table 2 Matrix of genetic distance (above diagonal) and geographic distance (below diagonal)

\begin{tabular}{lllllll}
\hline & GV10 & GV13 & GV18 & EH & RL & YK \\
\hline GV10 & 0 & 0.045 & 0.040 & 0.056 & 0.059 & 0.064 \\
GV13 & 0 & 0 & 0.057 & 0.063 & 0.067 & 0.068 \\
GV18 & 0 & 0 & 3.243 & 0.052 & 0.057 & 0.051 \\
EH & 3.243 & 3.547 & 3.547 & 2.526 & 0 & 0.054 \\
RL & 3.547 & 3.694 & 3.694 & 2.821 & 2.773 & 0 \\
YK & 3.694 & & & & 057 \\
\hline
\end{tabular}




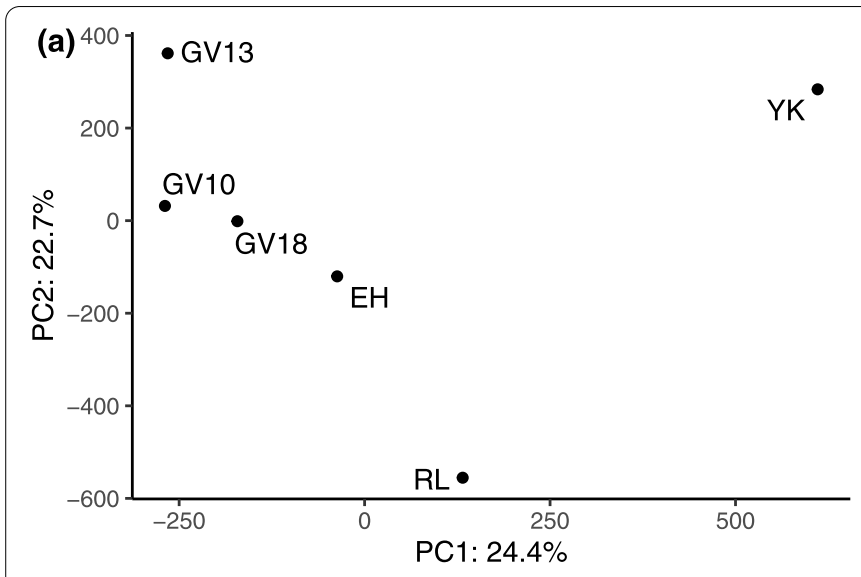

(b)

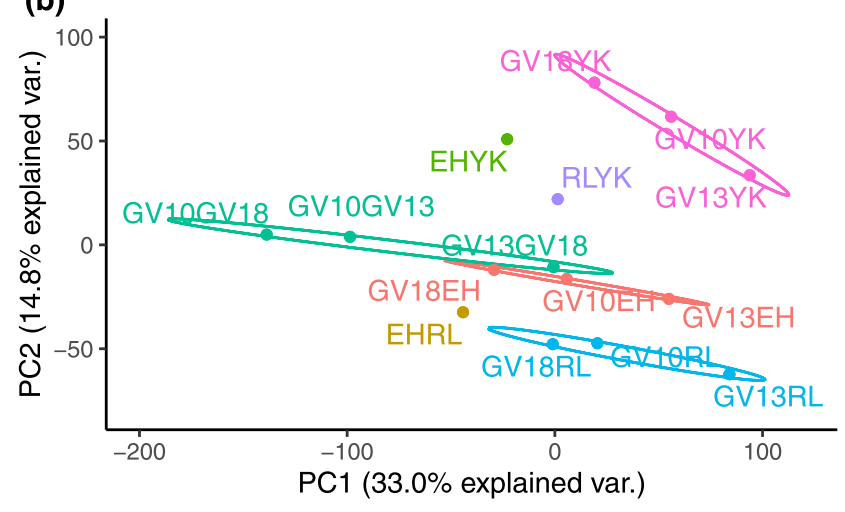

(d)

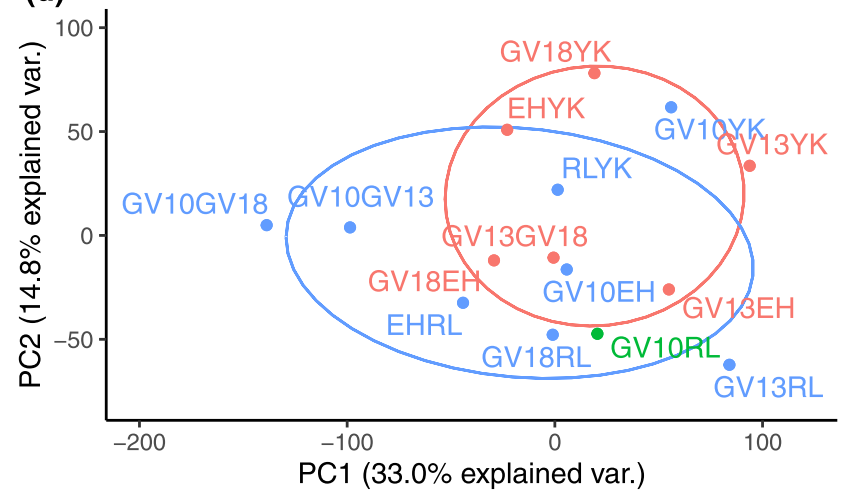

(c)

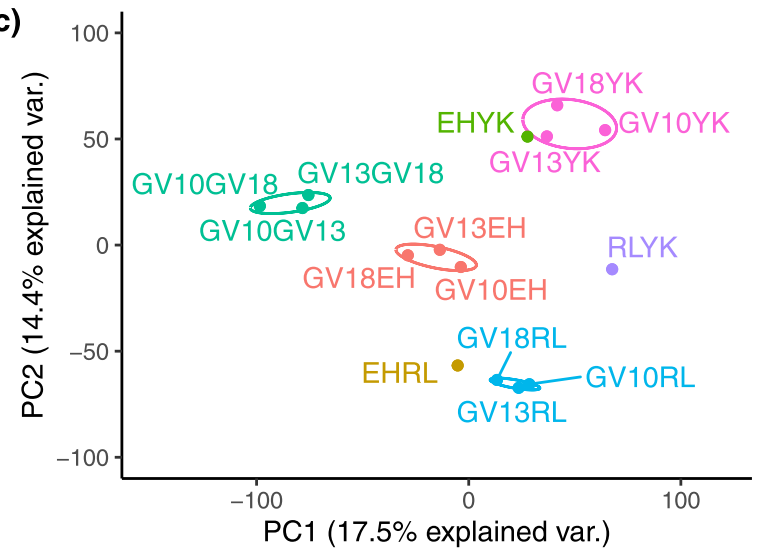

(e)

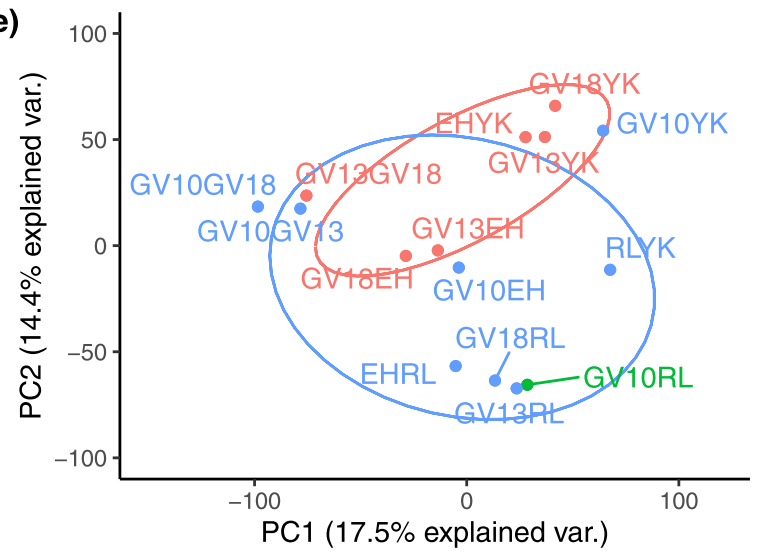

Fig. 4 Principal components analysis based on MAF or pairwise Fst. PCA plots of (a) allele frequency of Aedes aegypti samples (MAF $>0.1$, minimal coverage > 50); (b, d) pairwise Fst throughout the genome with $100 \mathrm{kbp}$ non-overlapping windows, and (c, e) pairwise Fst within genes. Colors in $(\mathbf{b}, \mathbf{c})$ represent geographical comparisons. The blue color in (d, e) represents comparisons between Wolbachia-infected and uninfected samples, while red represents comparisons within Wolbachia-infected samples and green represents the comparison within uninfected samples. The $95 \%$ confidence ellipses show data clustering

Pathway analysis and gene ontology enrichment analysis The "substantial" outliers were distributed within 187 genes (Additional file 2). When a region $50 \mathrm{kbp}$ upstream and $50 \mathrm{kbp}$ downstream around each outlier was considered, this number increased to 1436 genes of interest (Additional file 3). We performed a pathway analysis of these 1436 genes through the KEGG database [33] and found eight pathways significantly involved (Table 4, Additional file 4), including pathways involved with development and immune response (MAPK 


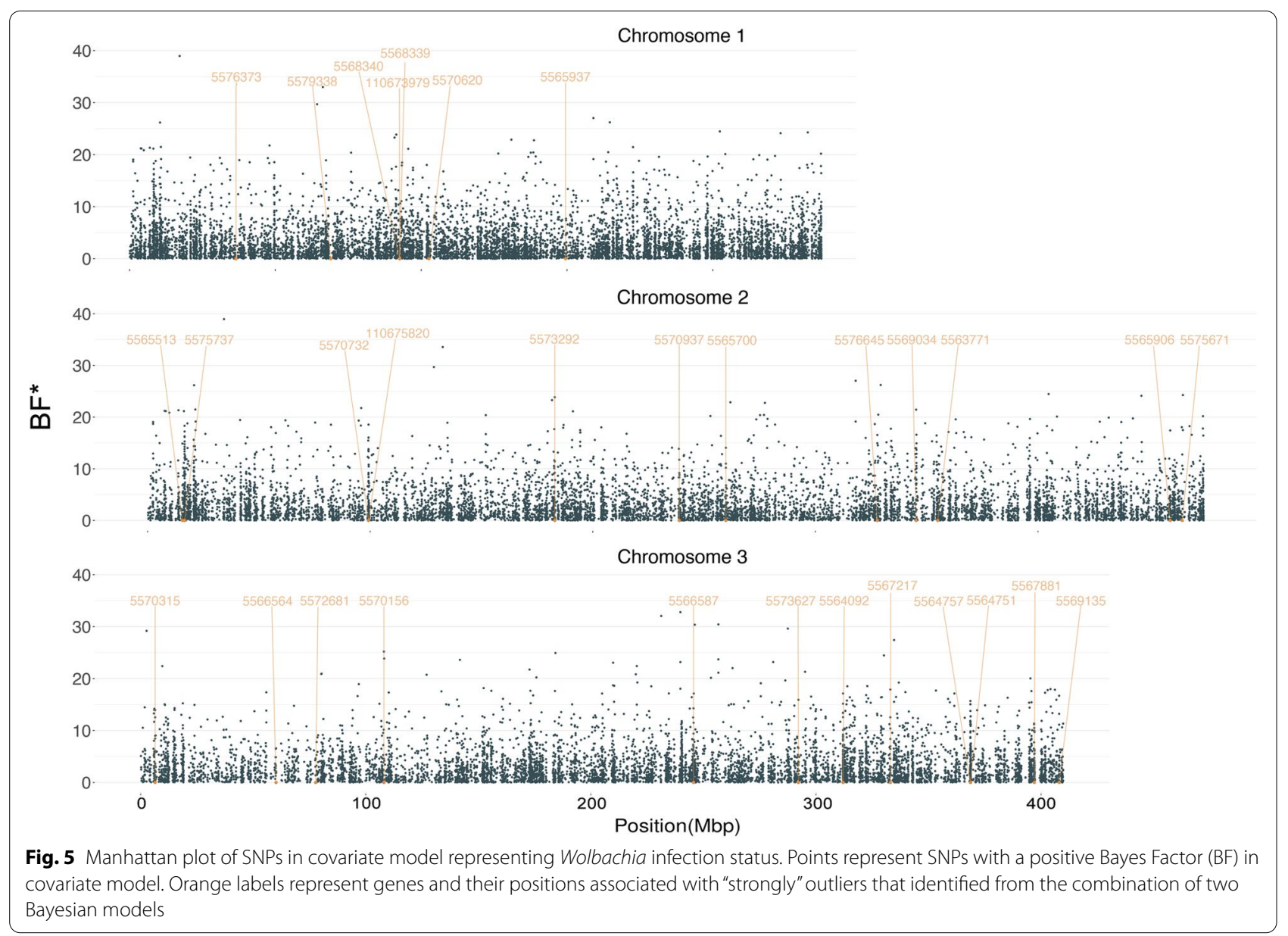

Table 3 Genomic regions potentially under Wolbachia wMel selection

\begin{tabular}{llll}
\hline Chromosome & Position & Size (Mbp) & $\begin{array}{l}\text { Number } \\
\text { of SNPs }\end{array}$ \\
\hline 1 & $96,900,000-105,500,000$ & 8.6 & 39 \\
1 & $138,000,000-140,500,000$ & 2.5 & 13 \\
1 & $148,500,000-149,500,000$ & 1.0 & 11 \\
1 & $183,000,000-188,300,000$ & 5.3 & 40 \\
1 & $203,100,000-206,200,000$ & 3.1 & 22 \\
2 & $15,600,000-16,800,000$ & 1.2 & 27 \\
2 & $20,700,000-21,200,000$ & 0.5 & 11 \\
2 & $98,800,000-99,300,000$ & 0.5 & 18 \\
2 & $345,200,000-345,400,000$ & 0.2 & 19 \\
2 & $396,100,000-396,500,000$ & 0.4 & 15 \\
3 & $239,700,000-249,400,000$ & 9.7 & 31 \\
3 & $311,900,000-316,000,000$ & 4.1 & 25 \\
3 & $368,400,000-368,600,000$ & 0.2 & 14 \\
\hline
\end{tabular}

signaling pathway and Toll and Imd signaling pathway). We also identified homologues of these genes in D. melanogaster for the gene ontology (GO) enrichment analysis, 108 gene sets were significantly enriched in biological process (Additional file 5), 42 in cellular component (Additional file 6) and 22 in molecular function (Additional file 7). In general, we found that Wolbachia $w \mathrm{Mel}$ may modulate genes with diverse functions such as cell development, interaction, cellular response, cellular transport, neurogenesis, lipid and glucide metabolization, behavior and immune response.

The "strong" outliers were distributed across 31 genes (Fig. 5, Additional file 1, Additional file 8). These included gene 5,564,751, which encodes cytochrome P450, which can be enriched in response to dengue virus infection in refractory mosquitoes [34] and is associated with insecticide resistance $[35,36]$. Carbonic anhydrase, encoded by gene $5,565,700$, has the function of balancing $\mathrm{pH}$ in mosquito midgut $[37,38]$. Gene 5,569,135 encoding ecdysone protein E75, and gene 5,572,681 encoding lipophorin, have been highly expressed in females after blood feeding and are potentially involved in regulation 
Table 4 Significantly enriched pathways in KEGG database potentially involved with Wolbachia wMel infection (copyright permission 210,807) [33]

\begin{tabular}{lllll}
\hline Pathway & Description & Gene Ratio & Bg Ratio & P value \\
\hline aag00232 & Caffeine metabolism & $6 / 296$ & $12 / 3281$ & $40 / 3281$ \\
aag00981 & Insect hormone biosynthesis & $9 / 296$ & $30 / 3281$ & $<.001$ \\
aag00052 & Galactose metabolism & $7 / 296$ & $25 / 3281$ & 0.008 \\
aag04341 & Hedgehog signaling pathway - fly & $6 / 296$ & $92 / 3281$ & 0.015 \\
aag04013 & MAPK signaling pathway - fly & $14 / 296$ & $96 / 3281$ & $30 / 3281$ \\
aag00230 & Purine metabolism & $14 / 296$ & $5 / 296$ & 0.034 \\
aag00270 & Cysteine and methionine metabolism & $9 / 296$ & $54 / 3281$ & 0.047 \\
aag04624 & Toll and Imd signaling pathway & & 0.050 \\
\hline
\end{tabular}

of oogenesis and vitellogenesis [39, 40]. Charged multivesicular body protein, encoded by gene $5,573,292$, is associated with endosome formation and can influence mosquito immune response [41].

\section{Discussion}

We show that Ae. aegypti populations in Cairns remain geographically distinct following releases of Wolbachia $w \mathrm{Mel}$, but also find some evidence suggesting evolutionary change in mosquito populations. When interpreting these results, it is important to consider the release process and which populations were targeted, and the fact that Wolbachia-induced CI can increase population divergence by reducing the migration rate across host populations when only one or both (in the case of different Wolbachia strains) are infected $[9,10]$. In release areas around Cairns, Gordonvale is relatively isolated from other release locations. Although these populations are not genetically isolated based on microsatellite and EPIC markers [42], they do appear to be somewhat separated based on the SNP markers used in the current study. This may account for the pattern noted for Tajima's D where the GV10 population was a clear outlier.

Following invasion by Wolbachia, there is not only complete replacement of the uninfected mosquito population by Wolbachia-infected mosquitoes but also replacement of the mtDNA that hitchhikes along with the Wolbachia [12]. Also, while any linkage disequilibrium between the Wolbachia and nuclear DNA variants is expected to break down relatively quickly [43], new alleles may nevertheless be introduced into the population. The nuclear DNA constitution of the population might be expected to become more like the release stock for a period as released females and their offspring mate with released and resident males, although local selection should then lead to populations becoming more like the original population. In our case, the genetic similarity among the Gordonvale samples before and after releases might reflect local selection and ongoing introgression of the release stock with the resident population, as GV10 and GV18 are closer than GV13 in the PCA analysis. Furthermore, Yorkeys Knob and Edge Hill remain distinct from each other despite previously being invaded by the same release stock [3, 7].

Populations were more differentiated at the gene level than at the genome level, which may be a consequence of a large proportion (47\%) of TEs and satellites associated with non-coding regions $[25,26]$. These TEs and satellites were masked in our reference genome [27], resulting in low coverage in sliding windows and were therefore filtered out in the gene analyses. This may explain why there was a high level of variation in the genome-wide scans of $\pi$ and Tajima's D. For chromosome 1, which contains the sex-determining regions, pools of male and female individuals were aligned to the reference genome, which can also result in low coverage of windows and induce high levels of genomic variation. Genic regions will be easier to track into the future for further analysis.

When considering the impacts of Wolbachia on the Ae. aegypti genome, selection in response to local conditions and the impact of $w \mathrm{Mel}$ on Ae. aegypti may only influence patterns of genetic differentiation at specific loci [19]. Our Bayesian outlier analysis identified several regions in each chromosome and genes related to immune response, development, recognition and behavior that may have been under selection. When overlapping these results with previous Tajima's D analysis, we identified only a small proportion of genes potentially related to selective sweeps, which were mainly distributed on chromosome 2. These potential evolutionary impacts of Wolbachia wMel on the genome of Ae. aegypti in the field suggest that further monitoring is warranted, although at this stage other factors unrelated to Wolbachia appear to have a larger impact on genomic differentiation among samples. 
We found signs of selection on Toll and Imd signaling pathways in KEGG analysis; these are important pathways in immune system [44, 45] and virus blocking processes [45-47]. Previous transcriptomic studies showed up-regulation of these pathways in both $w \mathrm{Mel}$ and $w \mathrm{Mel}-$ Pop-infected Ae. aegypti $[48,49]$. Genes associated with virus blocking are mainly distributed on chromosome 1 , in addition to genes associated with cytoskeleton, cellcell adhesion and signal transduction [19]; these genes also showed up in our GO enrichment analysis. Other than virus blocking, caffeine metabolism was strongly impacted, which may impact hormone metabolism and detoxification when cytochrome P450 is involved [50, 51]. We also detected enriched pathways involved with development, such as insect hormone biosynthesis and the Hedgehog signaling pathways. In the GO enrichment analysis these were represented in cell growth, structure, recognition and behavior.

In the past decade, the $w \mathrm{Mel}$ infection itself has not evolved in terms of either sequence composition or density since establishment in Ae. aegypti in northern Queensland, Australia [52]. Phenotypic comparisons also suggest limited changes in host fitness costs and CI since population replacement in this region $[14,18]$, although the number of fitness-related traits scored so far has been limited. Blockage of virus transmission also appears stable to date $[53,54]$, and may persist through ongoing selection favoring high viral blocking in Ae. aegypti populations [19]. However, the outlier loci detected here suggest that ongoing monitoring of phenotypic effects is warranted.

\section{Conclusions}

Wolbachia wMel-infected Ae. aegypti mosquitoes have been released successfully in the field to help reduce the transmission of arboviruses, but interactions between $w \mathrm{Mel}$ and Ae aegypti could result in adaptation $[55,56]$, altering virus blocking efficiency $[19,57]$, host fecundity [21] and insecticide resistance [58]. In this study, we have identified Ae. aegypti populations as being geographically distinct despite their Wolbachia infection status. However, selection associated with Wolbachia wMel may still have influenced variation at some loci. This is the first time that genome evolution associated with Wolbachia infection has been examined in field populations where there has been a deliberate release. However, it is hard to draw conclusions about long-term impacts of Wolbachia on the mosquito genome, which may take more time to develop, and which may be different in regions where dengue is endemic, unlike in Australia. Our findings highlight the possibility that the effect of Wolbachia can interact with the host genomic background, which has been shown previously in phenotypic assays of the longevity effects of wMelPop in Drosophila [59].

\section{Methods \\ Samples and study sites}

Aedes aegypti samples were collected from four sites around Cairns (Fig. 1, Table 1) [60]. In Gordonvale, samples were collected three times: in the summer of 2010 (pre-release), as well as in 2013 and 2018 (2 and 7 years post-release given that the area was stably invaded in 2011 [7]). Samples from Yorkeys Knob, Edge Hill and Redlynch were collected in 2018. Yorkeys Knob experienced Wolbachia invasion at the same time as Gordonvale in 2011, and Edge Hill which was invaded in 2013 [61]; Redlynch was an uninfected area when sampled in 2018. Gordonvale samples from 2010 and 2013 were collected by BG-Sentinel traps (Biogents, Regensburg, Germany) while 2018 samples were collected by ovitraps, taking care to sample only 1-2 larvae per ovitrap to reduce the likelihood of siblings being sampled [62]. Samples from Gordonvale 2010 (GV10) and 2013 (GV13) were stored in absolute ethanol at the adult stage while samples collected from 2018 (GV18, EH, YK and RL) were stored in absolute ethanol at the fourth instar larval stage.

\section{DNA extraction and library preparation}

Whole genomic DNA was extracted from each individual mosquito using Qiagen DNA Blood and Tissue kit (Venlo, Limburg, NL) for 2010 and 2013 samples, and using Roche High Pure ${ }^{\mathrm{TM}}$ PCR Template Preparation Kits (Roche Molecular Systems, Inc., Pleasanton, CA, USA) for 2018 samples. Wolbachia infection status was confirmed by a diagnostic qPCR test as outlined elsewhere [63]. The concentration of extracted individual DNA was measured using Quantitation Qubit ${ }^{\mathrm{TM}} 1 \mathrm{X}$ dsDNA HS Assay Kit (Invitrogen Life Technologies, USA). Samples from each of the six populations (Table 1) were pooled prior to sequencing based on an equal amount of DNA from each individual. Each population was sent for whole genome sequencing with $>50$ depth via Illumina Hiseq2500 using 100 bp paired read chemistry for GV10 and GV13, and $150 \mathrm{bp}$ paired read chemistry for GV18, EH, YK and RL libraries.

Raw sequences were trimmed using Trimmomatic v. 0.39 to truncate and remove low quality reads by requiring all reads to have all bases with a phred score $>20$ and read length $>70 \mathrm{bp}$. The reference genome AaegL5.0 [27] was indexed and reads were aligned using bowtie2 v. 2.3.4.3 with the --very-sensitive-local preset [64], with alignment rates ranging from 79.3 to $84.0 \%$. Samtools v.1.9 was used with default parameters to sort, mark and 
remove duplicates and generate pileup files requiring a minimal mapping quality of 20 .

\section{Estimation of genetic variation}

We investigated patterns of genetic variation within populations using PoPoolation v. 1.2.2 [65] with the genomic annotation file from the reference AaegL5.0 which has TEs and satellites masked. We calculated Tajima's pi (nucleotide diversity $\pi$ ) for each population at $10 \mathrm{kbp}$ non-overlapping windows and Tajima's D for each population at $10 \mathrm{kbp}$ non-overlapping windows and the gene level with a minimum coverage of 20 . Windows with low coverage generated no values and were omitted before adjusting the shape of lines across each chromosome by a LOESS smooth curve [66]. Within each retained window, at least $60 \%$ of sites had sufficient coverage (Additional file 9), indicating the high quality of all windows used for downstream analysis. The value of Tajima's D was calculated from allele frequencies in selected regions and was used to detect directionality of selection. Under a standard neutral model with no change in population size, a strongly negative Tajima's D value can indicate directional selection removing variation, while a strongly positive value can indicate balancing selection maintaining variation, with 0 reflecting an absence of selection.

For genetic variation between populations, we used PoPoolation2 v. 1.201 [67] to obtain allele frequency differences for each SNP. The SNPs were filtered to have a coverage $>50$ in all populations, ensuring that poorly sequenced areas were excluded. We also filtered to ensure an average MAF $>0.1$ [68]. We also obtained pairwise Fst values for non-overlapping $100 \mathrm{kbp}$ windows and for gene sets. The PCA was generated by the prcomp function and the ggbiplot package in R [69] based on: 1) MAF across the SNPs after initial filtering as mentioned above; 2) pairwise Fst values from $100 \mathrm{kbp}$ non-overlapping windows to indicate genetic distance patterns across the genome (genome level) [70]; and 3) pairwise Fst for genes (gene level). These Fst estimates were then used to assess patterns of similarity among samples with the same Wolbachia infection status in a pairwise comparison, and the same geographic distance in a pairwise comparison.

We further investigated IBD patterns among 2018 samples from the four locations by computing $\mathrm{Fst}^{*}=\mathrm{Fst} /$ (1-Fst) [71] based on the average pairwise Fst values from $100 \mathrm{kbp}$ non-overlapping windows. A geographic distance matrix was built based on the natural log transformation of the shortest road distance between the sampled locations as mosquito movement would be mostly by road transport [72]. We then looked for Fst patterns that might be related to this distance measure and ran a Mantel test through the ade4 package in $\mathrm{R}$ to test the relationship between genetic distance and geographic distance [73], with only 2018 populations included.

\section{Identification of outliers potentially associated with Wolbachia}

We used two models from a Bayesian outlier approach BayPass v. 2.2 [31] to identify outliers associated with Wolbachia infection. Firstly, we used a standard covariate model $[31,74]$, which requires a file providing values of each covariate to produce the Bayes factor (BF), the ratio of the likelihood of posterior and prior hypotheses, which can quantify the relative evidence of a candidate SNP being under selection [75]. BF were exported in $\mathrm{dB}$ units $\left(\mathrm{BF}^{*}=10 \times \log 10(\mathrm{BF})\right)$; the association with environmental variance was considered as "substantial" when $\mathrm{BF}^{*}>5$, "strongly-selected" when $\mathrm{BF}^{*}>10$ and "decisivelyselected" when $\mathrm{BF}^{*}>13$ following $\mathrm{H}$ Jeffreys [75]. We modelled Wolbachia infection status as a binary covariate by setting each infected sample as 1 and each uninfected sample as 0 . The measures of BF values are based on an Importance Sampling Approximation, which is unstable for single runs in particular when the number of population is small, so we averaged the BF values of three runs with different seeds from the random number generators following the suggestion in the manual of BayPass [31]. The introduction of linear relationships in this model, however, can lead to a high false positive rate from sampling noise, as the relationships of allele frequency among multiple populations are also influenced by other environmental factors, and are not independent from each other [32]. Based on this model, we generated plots for SNPs with average $\mathrm{BF}^{*}>0$ to illustrate the potential effects of the Wolbachia infection at the genome level; SNPs that could not be assigned a position on one of the three autosomes were discarded.

Secondly, we used a BayPass core model to identify outliers from comparisons between GV10 and GV13, GV10 and GV18, given that Gordonvale was the only location where we had samples at time points before and after the release. The infection of Wolbachia was considered as the main variable across time. An $\mathrm{X}^{\mathrm{T}} \mathrm{X}$ algorithm approach was used in this model, which was analogous to an Fst comparison [31, 32]. The $\mathrm{X}^{\mathrm{T}} \mathrm{X}$ value was used to identify selection pressure, with higher values suggesting positive selection and smaller values suggesting balancing selection [32]. In this model, we considered SNPs with $\mathrm{X}^{\mathrm{T}} \mathrm{X}$ values greater than the $90 \%$ threshold or $95 \%$ threshold in both the GV10 and GV13 comparison and the GV10 and GV18 comparison as candidates for intersecting with the "substantial" SNPs and "strongly-selected" SNPs from the first analysis. However, this model is unable to exclude the noise from gene flow or genetic drift due to the lack of duplicates, which can also cause false positives. We 
therefore considered the intersection of outliers from the above two models as SNPs potentially associated with Wolbachia infection. These SNPs were then matched with the GTF annotation file from NCBI to obtain a list of outlier genes.

\section{Pathway analysis and gene ontology enrichment analysis} We considered SNPs from the intersection of the two BayPass models above as outliers potentially under selection and searched the open reading frames to obtain a list of potentially important genes within a $100 \mathrm{kbp}$ region around each SNP (50 kbp upstream and $50 \mathrm{kbp}$ downstream) [76]. These genes were then searched through the KEGG pathway database [33] for interpretation. We then used BLAST+ (version 2.9.0) with the UniProtKB/ Swiss-Prot database [77] to identify homologous proteins in D. melanogaster. Only best matches were retained and further filtered with an e-value cut off $1.0 \mathrm{E}-10$ and $>60 \%$ identity $[78,79]$. Gene ontology (GO) enrichment analysis was then undertaken using the $\mathrm{R}$ packages clusterProfiler [80] and DOSE [81], with a false discovery rate cut-off of 0.05 after multiple hypothesis testing correction.

\section{Supplementary Information}

The online version contains supplementary material available at https://doi. org/10.1186/s12864-021-08200-1.

\section{Additional file 1.}

Additional file 2.

Additional file 3.

Additional file 4.

Additional file 5 .

Additional file 6.

Additional file 7.

Additional file 8.

Additional file 9.

\section{Acknowledgements}

We thank Gordana Rasic for her early contribution to the methodology for initial sequencing of the Gordonvale populations, and Moshe Jasper for his advice on data visualization. We also thank Scott Ritchie, Chris Paton and Mick Townsend from James Cook University for their support for sampling. This research was supported by use of the Nectar Research Cloud, a collaborative Australian research platform supported by the NCRIS-funded Australian Research Data Commons (ARDC).

\section{Authors' contributions}

AAH and TLS conceived the study. ML implemented the study, performed data analysis and wrote the first draft of the manuscript. All authors revised the manuscript. JC, QY and TLS provided guidance in data analysis. ML, TLS, $L S, Q Y$ and PAR contributed to sample preparation and/or sequencing. AAH secured financial support. All authors read and approved the final manuscript.

\section{Funding}

This research was funded by the National Health and Medical Research Council $(1132412,1118640$, www.nhmrc.gov.au). The funders had no role in the study design, data collection and analysis, decision to publish, or preparation of the manuscript.

\section{Availability of data and materials}

Aligned and merged sequencing data for Aedes aegypti used in this study are available from NCBI Sequence Read Archive (SRA), under the BioProject ID PRJNA776956. All data generated during this study are included within this article and its additional files.

\section{Declarations}

Ethics approval and consent to participate

Not applicable.

\section{Consent for publication}

Not applicable.

\section{Competing interests}

The authors declare that they have no competing interests.

\section{Author details}

${ }^{1}$ Pest and Environmental Adaptation Research Group, Bio21 Institute and the School of BioSciences, The University of Melbourne, Parkville, Victoria, Australia. ${ }^{2}$ Melbourne Bioinformatics, The University of Melbourne, Parkville, Victoria, Australia.

Received: 13 June 2021 Accepted: 15 November 2021

Published online: 14 December 2021

\section{References}

1. Nazni WA, Hoffmann AA, NoorAfizah A, Cheong YL, Mancini MV, Golding $\mathrm{N}$, et al. Establishment of Wolbachia strain wAlbB in Malaysian populations of Aedes aegypti for denque control. Curr Biol. 2019;29(24):4241-8.

2. Garcia GA, Sylvestre G, Aguiar R, da Costa GB, Martins AJ, Lima JBP, et al. Matching the genetics of released and local Aedes aegypti populations is critical to assure Wolbachia invasion. PLoS Negl Trop Dis. 2019;13(1):e0007023.

3. Ryan PA, Turley AP, Wilson G, Hurst TP, Retzki K, Brown-Kenyon J, Hodgson L, Kenny N, Cook H, Montgomery BL: Establishment of wMel Wolbachia in Aedes aegypti mosquitoes and reduction of local dengue transmission in Cairns and surrounding locations in northern Queensland, Australia. Gates Open Res 2019, 3.

4. Van den Hurk AF, Hall-Mendelin S, Pyke AT, Frentiu FD, McElroy K, Day A, et al. Impact of Wolbachia on infection with chikungunya and yellow fever viruses in the mosquito vector Aedes aegypti. PLoS Negl Trop Dis. 2012;6(11):e1892.

5. Aliota MT, Peinado SA, Velez ID, Osorio JE. The wMel strain of Wolbachia reduces transmission of Zika virus by Aedes aegypti. Sci Rep. 2016;6:28792.

6. Walker T, Johnson PH, Moreira LA, Iturbe-Ormaetxe I, Frentiu FD, McMeniman CJ, et al. The wMel Wolbachia strain blocks dengue and invades caged Aedes aegypti populations. Nature. 2011;476(7361):450-3.

7. Hoffmann AA, Montgomery B, Popovici J, Iturbe-Ormaetxe I, Johnson P, Muzzi F, et al. Successful establishment of Wolbachia in Aedes populations to suppress dengue transmission. Nature. 2011:476(7361):454-7.

8. Turelli M, Hoffmann AA. Cytoplasmic incompatibility in Drosophila simulans: dynamics and parameter estimates from natural populations. Genetics. 1995;140(4):1319-38.

9. Telschow A, Hammerstein P, Werren JH. Effects of Wolbachia on genetic divergence between populations: mainland-island model. Integr Comp Biol. 2002;42(2):340-51.

10. Telschow A, Hammerstein P, Werren JH. The effect of Wolbachia on genetic divergence between populations: models with two-way migration. Am Nat. 2002;160(Suppl 4):S54-66.

11. Kraaijeveld K, Franco P, de Knijff P, Stouthamer R, van Alphen JJ. Clonal genetic variation in a Wolbachia-infected asexual wasp: horizontal transmission or historical sex? Mol Ecol. 2011;20(17):3644-52.

12. Hale LR, Hoffmann AA. Mitochondrial DNA polymorphism and cytoplasmic incompatibility in natural populations of Drosophila simulans. Evolution. 1990;44(5):1383-6. 
13. Yeap HL, Rašić G, Endersby-Harshman NM, Lee SF, Arguni E, Le Nguyen $H$, et al. Mitochondrial DNA variants help monitor the dynamics of Wolbachia invasion into host populations. Heredity (Edinb). 2016;116(3):265-76.

14. Hoffmann AA, Iturbe-Ormaetxe I, Callahan AG, Phillips BL, Billington K, Axford JK, et al. Stability of the WMel Wolbachia infection following invasion into Aedes aegypti populations. PLoS Negl Trop Dis. 2014;8(9):e3115.

15. McMeniman CJ, Lane RV, Cass BN, Fong AWC, Sidhu M, Wang Y-F, et al. Stable introduction of a life-shortening Wolbachia infection into the mosquito Aedes aegypti. Science. 2009;323(5910):141-4.

16. Ross PA, Wiwatanaratanabutr I, Axford JK, White VL, Endersby-Harshman NM, Hoffmann AA: Wolbachia infections in Aedes aegypti differ markedly in their response to cyclical heat stress. PLoS Pathog 2017, 13(1):e1006006.

17. Bull JJ, Turelli M: Wolbachia versus dengue: evolutionary forecasts. Evol Med Public Health 2013, 2013(1):197-207.

18. Ross P, Hoffmann A. Continued susceptibility of the wMel Wolbachia infection in Aedes aegypti to heat stress following field deployment and selection. Insects. 2018;9(3):78.

19. Ford SA, Allen SL, Ohm JR, Sigle LT, Sebastian A, Albert I, et al. Selection on Aedes aegypti alters Wolbachia-mediated dengue virus blocking and fitness. Nat Microbiol. 2019;4(11):1832-9.

20. Ritchie SA, Townsend M, Paton CJ, Callahan AG, Hoffmann AA. Application of WMelPop Wolbachia strain to crash local populations of Aedes aegypti. PLoS Negl Trop Dis. 2015;9(7):e0003930.

21. Weeks AR, Turelli M, Harcombe WR, Reynolds KT, Hoffmann AA. From parasite to mutualist: rapid evolution of Wolbachia in natural populations of Drosophila. PLoS Biol. 2007;5(5):e114.

22. Hornett EA, Charlat S, Duplouy AM, Davies N, Roderick GK, Wedell N, et al. Evolution of male-killer suppression in a natural population. PLoS Biol. 2006;4(9):e283.

23. Carrington LB, Hoffmann AA, Weeks AR. Monitoring long-term evolutionary changes following Wolbachia introduction into a novel host: the Wolbachia popcorn infection in Drosophila simulans. Proc Biol Sci. 2010;277(1690):2059-68.

24. Ross PA, Turelli M, Hoffmann AA. Evolutionary ecology of Wolbachia releases for disease control. Annu Rev Genet. 2019;53:93-116.

25. Nene V, Wortman JR, Lawson D, Haas B, Kodira C, Tu ZJ, et al. Genome sequence of Aedes aegypti, a major arbovirus vector. Science. 2007;316(5832):1718-23.

26. Maringer K, Yousuf A, Heesom KJ, Fan J, Lee D, Fernandez-Sesma A, Bessant C, Matthews DA, Davidson AD: Proteomics informed by transcriptomics for characterising active transposable elements and genome annotation in Aedes aegypti. BMC Genomics 2017, 18(1):101-101.

27. Matthews BJ, Dudchenko O, Kingan SB, Koren S, Antoshechkin I, Crawford $J E$, et al. Improved reference genome of Aedes aegypti informs arbovirus vector control. Nature. 2018;563(7732):501-7.

28. Feschotte $C$. Transposable elements and the evolution of regulatory networks. Nat Rev Genet. 2008;9(5):397-405.

29. Lee Y, Schmidt H, Collier TC, Conner WR, Hanemaaijer MJ, Slatkin M, et al. Genome-wide divergence among invasive populations of Aedes aegypti in California. BMC Genomics. 2019;20(1):204.

30. Timoshevskiy VA, Kinney NA, Becky SD, Mao C, Tu Z, Severson DW, Sharakhov IV, Sharakhova MV: Genomic composition and evolution of Aedes aegypti chromosomes revealed by the analysis of physically mapped supercontigs. BMC Biol 2014, 12(1):27.

31. Gautier M. Genome-wide scan for adaptive divergence and association with population-specific covariates. Genetics. 2015;201(4):1555-79.

32. Günther T, Coop G. Robust identification of local adaptation from allele frequencies. Genetics. 2013;195(1):205-20.

33. Kanehisa M, Goto S. KEGG: Kyoto encyclopedia of genes and genomes. Nucleic Acids Res. 2000;28(1):27-30

34. Behura SK, Gomez-Machorro C, Harker BW, deBruyn B, Lovin DD, Hemme RR, et al. Global cross-talk of genes of the mosquito Aedes aegypti in response to dengue virus infection. PLoS Negl Trop Dis. 2011;5(11):e1385.

35. Bariami V, Jones CM, Poupardin R, Vontas J, Ranson H. Gene amplification, $A B C$ transporters and cytochrome P450s: unraveling the molecular basis of pyrethroid resistance in the dengue vector, Aedes aegypti. PLoS Negl Trop Dis. 2012;6(6):e1692.

36. Stevenson BJ, Pignatelli P, Nikou D, Paine MJ. Pinpointing P450s associated with pyrethroid metabolism in the dengue vector, Aedes aegypti: developing new tools to combat insecticide resistance. PLoS Negl Trop Dis. 2012;6(3):e1595.

37. Corena M, Seron TJ, Lehman HK, Ochrietor JD, Kohn A, Tu C, et al. Carbonic anhydrase in the midgut of larval Aedes aegypti: cloning, localization and inhibition. J Exp Biol. 2002;205(Pt 5):591-602.

38. del Pilar CM, VanEkeris L, Salazar MI, Bowers D, Fiedler MM, Silverman D, et al. Carbonic anhydrase in the adult mosquito midgut. J Exp Biol. 2005;208(Pt 17):3263-73.

39. Pierceall WE, Li C, Biran A, Miura K, Raikhel AS, Segraves WA. E75 expression in mosquito ovary and fat body suggests reiterative use of ecdysone-regulated hierarchies in development and reproduction. Mol Cell Endocrinol. 1999;150(1-2):73-89.

40. van Heusden MC, Thompson F, Dennis J. Biosynthesis of Aedes aegypti lipophorin and gene expression of its apolipoproteins. Insect Biochem Mol Biol. 1998;28(10):733-8.

41. Bartholomay LC, Cho WL, Rocheleau TA, Boyle JP, Beck ET, Fuchs JF, et al. Description of the transcriptomes of immune response-activated hemocytes from the mosquito vectors Aedes aegypti and Armigeres subalbatus. Infect Immun. 2004;72(7):4114-26.

42. Endersby NM, Hoffmann AA, White VL, Lowenstein S, Ritchie S, Johnson PH, et al. Genetic structure of Aedes aegypti in Australia and Vietnam revealed by microsatellite and exon primed intron crossing markers suggests feasibility of local control options. J Med Entomol. 2009;46(5):1074-83.

43. Turelli M, Hoffmann AA, McKechnie SW. Dynamics of cytoplasmic incompatibility and mtDNA variation in natural Drosophila simulans populations. Genetics. 1992;132(3):713-23.

44. Waterhouse RM, Kriventseva EV, Meister S, Xi Z, Alvarez KS, Bartholomay LC, et al. Evolutionary dynamics of immune-related genes and pathways in disease-vector mosquitoes. Science. 2007;316(5832):1738-43.

45. Pan X, Pike A, Joshi D, Bian G, McFadden MJ, Lu P, et al. The bacterium Wolbachia exploits host innate immunity to establish a symbiotic relationship with the dengue vector mosquito Aedes aegypti. The ISME journal. 2018;12(1):277-88.

46. Xi Z, Ramirez JL, Dimopoulos G: The Aedes aegypti toll pathway controls dengue virus infection. PLoS Pathog 2008, 4(7):e1000098-e1000098.

47. Kambris Z, Blagborough AM, Pinto SB, Blagrove MSC, Godfray HCJ, Sinden RE, Sinkins SP: Wolbachia stimulates immune gene expression and inhibits plasmodium development in Anopheles gambiae. PLoS Pathog 2010, 6(10):e1001143-e1001143.

48. Kambris Z, Cook PE, Phuc HK, Sinkins SP. Immune activation by lifeshortening Wolbachia and reduced filarial competence in mosquitoes. Science. 2009;326(5949):134-6.

49. Rancès $\mathrm{E}$, Ye YH, Woolfit M, McGraw EA, O'Neill SL. The relative importance of innate immune priming in Wolbachia-mediated dengue interference. PLoS Pathog. 2012;8(2):e1002548.

50. Coelho A, Fraichard S, Le Goff G, Faure P, Artur Y, Ferveur JF, et al. Cytochrome P450-dependent metabolism of caffeine in Drosophila melanogaster. PLoS One. 2015;10(2):e0117328.

51. Chung H, Sztal T, Pasricha S, Sridhar M, Batterham P, Daborn PJ. Characterization of Drosophila melanogaster cytochrome P450 genes. Proc Natl Acad Sci U S A. 2009;106(14):5731-6.

52. Huang $B$, Yang $Q$, Hoffmann AA, Ritchie $S A$, van den Hurk AF, Warrilow D: Wolbachia genome stability and mtDNA variants in Aedes aegypti field populations eight years after release. iscience 2020:101572.

53. Frentiu FD, Zakir T, Walker T, Popovici J, Pyke AT, van den Hurk A, McGraw EA, O'Neill SL: Limited dengue virus replication in field-collected Aedes aegypti mosquitoes infected with Wolbachia. PLoS Negl Trop Dis 2014, $8(2)$.

54. Carrington LB, Tran BCN, Le NTH, Luong TTH, Nguyen TT, Nguyen PT, et al. Field- and clinically derived estimates of Wolbachia-mediated blocking of dengue virus transmission potential in Aedes aegypti mosquitoes. Proc Natl Acad Sci U S A. 2018;115(2):361-6.

55. McGraw E, Merritt D, Droller J, O'Neill SL: Wolbachia density and virulence attenuation after transfer into a novel host. Proc Natl Acad Sci U S A 2002, 99(5):2918-2923.

56. Turelli M. Evolution of incompatibility-inducing microbes and their hosts. Evolution. 1994;48(5):1500-13.

57. Terradas G, Allen SL, Chenoweth SF, McGraw EA. Family level variation in Wolbachia-mediated dengue virus blocking in Aedes aegypti. Parasit Vectors. 2017:10(1):622. 
58. Echaubard P, Duron O, Agnew P, Sidobre C, Noël V, Weill M, et al. Rapid evolution of Wolbachia density in insecticide resistant Culex pipiens. Heredity (Edinb). 2010;104(1):15-9.

59. Carrington LB, Leslie J, Weeks AR, Hoffmann AA. The popcorn Wolbachia infection of Drosophila melanogaster: can selection alter i longevity effects? Evolution. 2009;63(10):2648-57.

60. Kahle DJ, Wickham H. Ggmap: spatial visualization with ggplot2. R J. 2013;5(1):144

61. Schmidt TL, Barton NH, Rašić G, Turley AP, Montgomery BL, IturbeOrmaetxe I, et al. Local introduction and heterogeneous spatial spread of dengue-suppressing Wolbachia through an urban population of Aedes aegypti. PLoS Biol. 2017;15(5):e2001894.

62. Jasper M, Schmidt TL, Ahmad NW, Sinkins SP, Hoffmann AA: A genomic approach to inferring kinship reveals limited intergenerational dispersal in the yellow fever mosquito. Mol Ecol Resour 2019.

63. Lee SF, White VL, Weeks AR, Hoffmann AA, Endersby NM. High-throughput PCR assays to monitor Wolbachia infection in the dengue mosquito (Aedes aegypti) and Drosophila simulans. Appl Environ Microbiol. 2012;78(13):4740-3.

64. Langmead B, Salzberg SL. Fast gapped-read alignment with bowtie 2. Nat Methods. 2012:9(4):357-9.

65. Kofler R, Orozco-terWengel P, De Maio N, Pandey RV, Nolte V, Futschik A, Kosiol C, Schlötterer C: PoPoolation: a toolbox for population genetic analysis of next generation sequencing data from pooled individuals. PLoS One 2011, 6(1):e15925-e15925.

66. Wickham H: ggplot2: elegant graphics for data analysis: Springer; 2016.

67. Kofler R, Pandey RV, Schlötterer C. PoPoolation2: identifying differentiation between populations using sequencing of pooled DNA samples (Pool-Seq). Bioinformatics. 2011;27(24):3435-6.

68. Asif H, Alliey-Rodriguez N, Keedy S, Tamminga CA, Sweeney JA, Pearlson G, et al. GWAS significance thresholds for deep phenotyping studies can depend upon minor allele frequencies and sample size. Mol Psychiatry. 2020

69. Team RC. R: a language and environment for statistical computing. Vienna: R Foundation for Statistical Computing; 2019.

70. Hivert V, Leblois R, Petit EJ, Gautier M, Vitalis R. Measuring genetic differentiation from pool-seq data. Genetics. 2018;210(1):315-30.

71. Rousset F. Genetic differentiation and estimation of gene flow from F-statistics under isolation by distance. Genetics. 1997;145(4):1219-28.

72. Medley KA, Jenkins DG, Hoffman EA. Human-aided and natural dispersal drive gene flow across the range of an invasive mosquito. Mol Ecol. 2015;24(2):284-95.

73. Diniz-Filho JAF, Soares TN, Lima JS, Dobrovolski R, Landeiro VL, de Campos Telles MP, et al. Mantel test in population genetics. Genet Mol Biol. 2013;36(4):475-85.

74. Coop G, Witonsky D, Di Rienzo A, Pritchard JK. Using environmental correlations to identify loci underlying local adaptation. Genetics. 2010;185(4):1411-23.

75. Jeffreys H. The theory of probability. Oxford.: Oxford University Press; 1961

76. Quinlan AR, Hall IM. BEDTools: a flexible suite of utilities for comparing genomic features. Bioinformatics. 2010;26(6):841-2.

77. Camon E, Magrane M, Barrell D, Lee V, Dimmer E, Maslen J, Binns D, Harte N, Lopez R, Apweiler R: The gene ontology annotation (Goa) database: sharing knowledge in uniprot with gene ontology. Nucleic Acids Res 2004;32(suppl_1):D262-D266.

78. Pearson WR: An introduction to sequence similarity ("homology") searching. Curr Protoc Bioinformatics 2013, Chapter 3:Unit3.1.

79. Tian W, Skolnick J. How well is enzyme function conserved as a function of pairwise sequence identity? J Mol Biol. 2003;333(4):863-82.

80. Yu G, Wang L-G, Han Y, He Q-Y. clusterProfiler: an R package for comparing biological themes among gene clusters. OMICS. 2012;16(5):284-7.

81. Yu G, Wang L-G, Yan G-R, He Q-Y. DOSE: an R/Bioconductor package for disease ontology semantic and enrichment analysis. Bioinformatics. 2015;31(4):608-9.

\section{Publisher's Note}

Springer Nature remains neutral with regard to jurisdictional claims in published maps and institutional affiliations.

Ready to submit your research? Choose BMC and benefit from:

- fast, convenient online submission

- thorough peer review by experienced researchers in your field

- rapid publication on acceptance

- support for research data, including large and complex data types

- gold Open Access which fosters wider collaboration and increased citations

- maximum visibility for your research: over 100M website views per year

At BMC, research is always in progress.

Learn more biomedcentral.com/submissions 